\title{
Detectability of cosmic topology in generalized Chaplygin gas models
}

\author{
B. $\operatorname{Mota}^{1}$, M. Makler ${ }^{1,2,3}$, and M. J. Rebouças ${ }^{1}$ \\ 1 Centro Brasileiro de Pesquisas Físicas, Rua Dr. Xavier Sigaud, 150, 22290-180 Rio de Janeiro - RJ, Brazil \\ e-mail: [brunom;reboucas;martin]@cbpf.br \\ 2 Universidade Federal do Rio de Janeiro, Instituto de Física, CP 68528, 21945-972 Rio de Janeiro - RJ, Brazil \\ 3 Observatório Nacional - MCT, Rua Gal. José Cristino, 77 20921-400, Rio de Janeiro - RJ, Brazil
}

Received 17 April 2005 / Accepted 13 September 2005

\section{ABSTRACT}

If the spatial section of the universe is multiply connected, repeated images or patterns are expected to be detected in observations. However, due to the finite distance to the last scattering surface, such repeated patterns could be unobservable. This raises the question of whether a given cosmic topology is detectable, depending on the values of the parameters of the cosmological model. We study how the detectability is affected by the choice of the model itself for the matter-energy content of the universe, focusing our attention on the generalized Chaplygin gas (GCG) model for dark matter and dark energy unification, and investigate how the detectability of cosmic topology depends on the GCG parameters. We determine to what extent a number of topologies is detectable for the current observational bounds on these parameters. It emerges from our results that the choice of the GCG as an alternative to the $\Lambda$ CDM matter-energy content model has an impact on the detectability of cosmic topology. In particular more topologies become detectable for a certain range of the GCG parameters. We stress that the method described here can be applied to any model for the matter-energy content.

Key words. cosmology: dark matter - large-scale structure of Universe - cosmological parameters

\section{Introduction}

Recent advances in observational cosmology have put stringent constraints on the kind of universe we live in. Briefly, evidence points to a cosmos which is homogeneous on large scales, which has spatial curvature close to zero, and where baryonic matter and radiation contribute to only about $5 \%$ of the its total density. The bulk of the universe's matter-energy content seems to be composed of a pressureless component that is responsible for the observed clustering of luminous matter (accounting for almost a third of the universe's total density) and of a negative pressure component that drives the present phase of accelerated expansion (responsible for the remaining two thirds of the total density). Neither of these components can be directly observed, and they are usually referred to as dark matter (DM) and dark energy (DE).

It has been proposed that both DE and DM may be described by a single fluid, reducing to one the unknown components of the material substratum of the universe. Among the candidates for such unifying DM are the Chaplygin gas and its generalizations (see for example Kamenshchik et al. 2001; Makler 2001; Bilić et al. 2002). At high densities, such as those found at redshifts $z \gg 1$ or in galaxy clusters, this component behaves as DM, while at low densities (such as the average density at $z \lesssim 1$ ), its pressure becomes increasingly negative, thereby explaining the current accelerated expansion of the universe. Observational data constrain the Chaplygin model's ${ }^{1}$ parameter space, but does not rule it out (see, e.g., Makler et al. 2003b; Reis et al. 2003; Dev et al. 2004; Zhu 2004, and references therein).

In a hitherto unrelated line of research, a great deal of work has recently gone into studying the possibility that the universe may possess compact spatial sections with a non-trivial topology (for reviews, see for instance Lachièze-Rey et al. 1995; Levin 2002; Rebouças \& Gomero 2004). Many methods of detecting such topology have been proposed (see, e.g., Lehoucq et al. 1998; Cornish et al. 1996; Uzan et al. 1999), while the increasing accuracy of observational cosmology now makes it possible to apply these methods to existing observational data (see, e.g., Luminet et al. 2003; Cornish et al. 2004; de Oliveira-Costa et al. 2004; Roukema et al. 2004).

A direct way of determining the existence of a multiply connected 3-space section $M$ is to detect repeated images of radiating sources, which implies that the separation between correlated pairs must be smaller than twice the radius of the

${ }^{1}$ We note that the Chaplygin gas cosmological solution can also be mimicked by a classical scalar field (Kamenshchik et al. 2001), although the initial value of the field must be extremely fine-tuned, see Perrotta et al. (2004). 
observable universe, $d_{\text {hor }}$. Note, however, that this distance between images, being the length of the associated closed geodesic, is fixed for each nonflat topology in units of the curvature radius $a_{0}$. As a consequence, many possible topologies may be undetectable by repetition of patterns, given the near flatness of the universe favored by current observations (see, e.g., Tegmark et al. 2004). Thus, the ratio $\chi_{\text {hor }}=d_{\text {hor }} / a_{0}$, which depends on the cosmological model and its associated parameters, is crucial in determining the detectability of cosmic topology.

The extent to which a nontrivial topology may or may not be detected for the current bounds on the cosmological parameters has been studied in Gomero et al. (2001a,b), Mota et al. (2003), Weeks (2003), and Weeks et al. (2003). These studies have concentrated on cases where the matter-energy content is modelled within the $\Lambda \mathrm{CDM}$ framework, by investigating the dependence of the detectability of the topology on the cosmological density parameters $\Omega_{\mathrm{m}}$ and $\Omega_{\Lambda}$. Here we address a different question: to what extent is the detectability of the topology modified when different background cosmological models are considered? To this end, we focus our attention on lowcurvature nonflat universes whose dark-matter-energy content is dominated by the generalized Chaplygin Gas (GCG). We determine which topologies from a large family of spherical manifolds and some small-volume hyperbolic manifolds are either potentially detectable or undetectable, taking current observational bounds on the GCG model into account. We also study the dependence of detectability on the model parameters and, in particular, on the steepness of the equation of state, $\alpha$, and show that detectability becomes more likely as $\alpha$ decreases.

This paper is organized as follows. In the next section we discuss some fundamental results on the detectability of cosmic topology and provide a short review of GCG-dominated cosmological models. These two strands are brought together in Sect. 3, which deals with our analytical and numerical results regarding the detectability of cosmic topology in the GCG case. Finally, we sum up our results and indicate points for future research in Sect. 4.

\section{Preliminaries}

Most cosmologists agree that the universe is close to being spatially homogeneous and isotropic on large scales. In a general relativistic context, such space-time is described by a 4-manifold $\mathcal{M}$ that is decomposed into $\mathcal{M}=\mathbb{R} \times M$ and is endowed with a Robertson-Walker metric

$\mathrm{d} s^{2}=-\mathrm{d} t^{2}+a^{2}(t)\left[\mathrm{d} \chi^{2}+f^{2}(\chi)\left(\mathrm{d} \theta^{2}+\sin ^{2} \theta \mathrm{d} \phi^{2}\right)\right]$,

where $a(t)$ is the scale factor and $f(\chi)=\chi, \sin \chi$, or $\sinh \chi$, depending on the sign of the curvature (respectively $k=0,1$, or -1$)$. For each of these three cases, the spatial section $M$ is usually taken to be a simply-connected manifold, either Euclidian $\mathbb{E}^{3}(k=0)$, spherical $\mathbb{S}^{3}(k=1)$, or hyperbolic $\mathbb{H}^{3}$ $(k=-1)$. However, since geometry does not dictate topology, the 3-space $M$ may as well be any one of a number of (multiply connected) quotient manifolds $M=\widetilde{M} / \Gamma$, where $\Gamma$ is a discrete and fixed point-free group of isometries of the covering space $\widetilde{M}=\left(\mathbb{E}^{3}, \mathbb{S}^{3}, \mathbb{H}^{3}\right)$. The action of $\Gamma$ tessellates $\widetilde{M}$ into identical domains that are copies of what is known as the fundamental polyhedron. Hence, a point $x$ (or source) within the fundamental polyhedron can be connected to every other point on its orbit in the covering space $\left.g x\right|_{g \in \Gamma}$ (i.e., its images) by a space-like geodesic, which is closed by the associated isometry. The immediate observational consequence of this is that the sky may (potentially) show multiple images of radiating sources. The closed geodesics are the projection onto $M$ of the (light-like) geodesics followed by photons emitted by the source. By observing repeated images or patterns, we are directly detecting isometries of the group $\Gamma$ with which we might reconstruct the topology of $M$.

A closed geodesic that passes through $x$ in a multiply connected manifold $M$ is a segment of a geodesic in the covering space $\widetilde{M}$ that joins $x$ to one of its images $\left.g x\right|_{g \in \Gamma}$. Since any such pair of points is related by an isometry $g \in \Gamma$, the length of the closed geodesic associated to any fixed isometry $g$ passing through $x$ is given by the corresponding distance function $\delta_{g}(x) \equiv d(x, g x)$. The injectivity radius at $x$ is then defined as

$r_{\mathrm{inj}}(x) \equiv \frac{1}{2} \min _{g \in \Gamma, g \neq I}\left\{\delta_{g}(x)\right\}$,

where $I$ is the identity of $\Gamma$. From (2) the injectivity radius is clearly the radius of the smallest sphere centered at $x$ and inscribable in the fundamental polyhedron. Thus, any sphere with radius $r<r_{\text {inj }}(x)$ and centered at $x$ lies inside a fundamental polyhedron of $M$ based on $x$.

One can also define the global injectivity radius (simply denoted by $r_{\text {inj }}$ ), which is the radius of the smallest sphere inscribable in $M$ whose radius clearly is the minimum value of $r_{\text {inj }}(x)$

$r_{\mathrm{inj}} \equiv \min _{x \in M}\left\{r_{\mathrm{inj}}(x)\right\}$.

Due to the finite age of the universe, it is generally not possible to observe the entire covering space. For any given observational survey up to a maximum redshift $z_{\text {obs }}$, the survey depth $\chi\left(z_{\mathrm{obs}}, \Omega_{i 0}\right)$ in a nonflat universe can be expressed in units of the curvature radius ${ }^{2} a_{0}=\left(H_{0} \sqrt{\left|1-\Omega_{0}\right|}\right)^{-1}$, as a function of the (present time) density parameters $\Omega_{i 0}=\rho_{i 0} / \rho_{\text {crit }}$, as

$\chi\left(z_{\mathrm{obs}}, \Omega_{i 0}\right)=\frac{d_{\mathrm{hor}}}{a_{0}}=\sqrt{\left|1-\Omega_{0}\right|} \int_{0}^{z_{\mathrm{obs}}} \frac{H_{0}}{H\left(z, \Omega_{i 0}\right)} \mathrm{d} z$,

where $H\left(z, \Omega_{i 0}\right)$ is the Hubble parameter at redshift $z$.

Now, for a given $\chi\left(z_{\mathrm{obs}}, \Omega_{i 0}\right)$ (or simply $\left.\chi_{\mathrm{obs}}\right)$, a topology is undetectable by an observer at a point $x$ if $\chi_{\mathrm{obs}}<r_{\mathrm{inj}}(x)^{3}$. In this case there are no multiple images (or repeated patterns) in the survey. On the other hand, when $\chi_{\mathrm{obs}}>r_{\mathrm{inj}}(x)$, then the topology is detectable in principle for an observer at $x$. However, since we do not know a priori our position in the universe, it is more suitable for our purposes here to use a criterium valid at any $x$, stated in terms of the global injectivity radius $r_{\text {inj }}$, which is a lower bound for $r_{\text {inj }}(x)$. The undetectability condition then is that the topology of $M$ is undetectable by any observer if $r_{\text {inj }}>\chi_{\text {obs. }}$. Reciprocally, the condition $\chi_{\text {obs }}>r_{\text {inj }}$ ensures

2 Here $H_{0}$ is the Hubble constant, and $\Omega_{0}=\rho_{0} / \rho_{\text {crit }}$ is the total density parameter with $\rho_{\text {crit }}=3 H_{0}^{2} / 8 \pi G$.

3 In this work we express $r_{\text {inj }}$ in units of $a_{0}$. 
Table 1. Single action spherical manifolds, along with the order of the covering groups and the injectivity radii.

\begin{tabular}{lcc}
\hline \hline Name and symbol & Order of $\Gamma$ & Injectivity radius \\
\hline Cyclic $Z_{n}$ & $n$ & $\pi / n$ \\
Binary dihedral $D_{m}^{*}$ & $4 m$ & $\pi / 2 m$ \\
Binary tetrahedral $T^{*}$ & 24 & $\pi / 6$ \\
Binary octahedral $O^{*}$ & 48 & $\pi / 8$ \\
Binary icosahedral $I^{*}$ & 120 & $\pi / 10$ \\
\hline
\end{tabular}

detectability in principle for at least some observers. In globally homogeneous manifolds, the distance function $d(x, g x)$ for any given isometry $g$ is constant, which means $r_{\mathrm{inj}}(x) \equiv r_{\mathrm{inj}}$. Therefore, if the topology is potentially detectable (or conversely, undetectable) by an observer at $x$, it is likewise potentially detectable (conversely undetectable) by any other observer at any other point in the 3 -space $M$.

We focus exclusively on the detectability of spherical and hyperbolic manifolds. These manifolds are rigid, which implies that geometrical quantities are topological invariants and thus that the lengths of the closed geodesics are fixed in units of the curvature radius $a_{0}$. We can therefore compare the injectivity radius $r_{\text {inj }}$ of each manifold with the survey depth $\chi_{\text {obs }}$ as a function of the matter-energy content model parameters.

The spherical 3-manifolds have the form $M=\mathbb{S}^{3} / \Gamma$, where $\Gamma$ is a finite subgroup of $S O(4)$ acting freely on the 3 -sphere. These manifolds were originally classified by Threlfall and Seifert (1932), and are also discussed by Wolf (1984); for a description in the context of cosmic topology, see Ellis (1971). This classification essentially consists in enumerating all finite groups $\Gamma \subset S O(4)$, and then grouping all manifolds in classes. In a recent paper, Gausmann et al. (2001) recast the classification in terms of single action, double action, and linked action manifolds. In Table 1 we list the orientable single action manifolds together with the labels often used to refer to them, along with the order of the covering groups $\Gamma$ and the corresponding injectivity radii. All single action manifolds are globally homogeneous, and thus the same detectability condition holds for all observers in the manifold.

Closed orientable hyperbolic 3-manifolds can be constructed and studied with the publicly available SnapPea software package (Weeks 1999). Each compact hyperbolic manifold is constructed from a non-compact cusped manifold through so-called Dehn surgery, which is a formal procedure identified by two coprime integers called winding numbers $\left(n_{1}, n_{2}\right)$. SnapPea names these manifolds according to the original cusped manifold and the winding numbers, so, for example, the smallest volume hyperbolic manifold known (the Weeks' manifold) is labelled as $\mathrm{m003}(-3,1)$, where $\mathrm{m003}$ is the seed cusped manifold and $(-3,1)$ are the winding numbers. Hodgson and Weeks (1994) have compiled a census of 11031 orientable closed hyperbolic 3-manifolds ordered by increasing volume. In Table 2 we present the seven smallest manifolds from this census, with their respective volumes and injectivity radii $r_{\text {inj. }}$.

It is clear from Eq. (4) that, since the integral is finite, more and more nonflat topologies become undetectable as $\Omega_{0} \rightarrow 1$.
Table 2. First seven manifolds in the Hodgson-Weeks census of closed hyperbolic manifolds, with corresponding volumes and injectivity radii.

\begin{tabular}{lcc}
\hline \hline Manifold & Volume & Injectivity radius \\
\hline $\mathrm{m} 003(-3,1)$ & 0.943 & 0.292 \\
$\mathrm{~m} 003(-2,3)$ & 0.981 & 0.289 \\
$\mathrm{~m} 007(3,1)$ & 1.015 & 0.416 \\
$\mathrm{~m} 003(-4,3)$ & 1.264 & 0.287 \\
$\mathrm{~m} 004(6,1)$ & 1.284 & 0.240 \\
$\mathrm{~m} 004(1,2)$ & 1.398 & 0.183 \\
$\mathrm{~m} 009(4,1)$ & 1.414 & 0.397 \\
\hline
\end{tabular}

To have a quantitative estimate of detectability, however, one must specify the matter-energy models in order to have the Hubble function $H\left(z, \Omega_{i 0}\right)$ (cf. Eq. (4)). Therefore, the choice of model for the matter-energy content of the universe has, as we discuss below, important consequences for the possible detection of cosmic topology. In the present work we study the detectability of cosmic topology in a universe dominated by the GCG, which we now review briefly.

As mentioned in the introduction, the dynamics of the universe seems to be dominated by two components that are not directly observable: a pressureless DM component and a DE component with negative pressure. DM is detected by its local clustering, through gravitational lensing and by dynamical probes, such as galaxy rotation curves, velocity dispersion, and $X$-ray emission by gas in clusters. The observed abundance of light elements, together with primordial nucleosynthesis calculations, shows that baryons can account for only about $15 \%$ of this clustered component (see, e.g., Bertone et al. 2004). On the other hand, the presence of DE is made evident by its effects on very large scales. It seems to power the accelerated expansion discovered in type Ia supernovae (SNIa) data (see, e.g., Perlmutter et al. 1998; Riess et al. 1998; Tonry et al. 2003) and to significantly contribute to the global curvature, providing around two thirds of the density needed to explain the near flatness inferred from the cosmic microwave background radiation (CMBR) data.

Since the evidence for DM and DE involves observations at different scales and epochs, one may wonder if they could be distinct manifestations of the same component, dubbed unifying dark matter, or UDM (see, e.g., Makler et al. 2003a). We assume that the UDM may be modelled by a perfect fluid energymomentum tensor whose only independent components are $p$ and $\rho$. The UDM equation of state must allow for a decelerating and non-relativistic phase in the past, followed by the present accelerated phase. Recall that the acceleration is given by $\ddot{a}=-4 \pi G(\rho+3 p) / 3$. Thus, for the decelerating and accelerating phases to happen sequentially the UDM must be nearly pressureless at higher densities, with $|p| \ll \rho$, and also exert a large $(p \sim-\rho)$ negative pressure at lower densities. A simple example of an equation of state satisfying this condition is that of the GCG (see Makler 2001; Bilić et al. 2002; Bento et al. 2002), and is given by an inverse power law

$p_{\mathrm{Ch}}=-\frac{M^{4(\alpha+1)}}{\rho_{\mathrm{Ch}}^{\alpha}}$, 
where $M$ is positive and has the dimension of mass, and $\alpha$ is an adimensional real number.

Consider now the background geometry of a universe dominated by the GCG. In this case, the energy conservation equation can be solved easily, giving the energy density of the GCG in terms of the scale factor,

$\rho_{\mathrm{Ch}}=\rho_{\mathrm{Ch} 0}\left[(1-A)\left(\frac{a_{0}}{a}\right)^{3(\alpha+1)}+A\right]^{1 /(\alpha+1)}$,

where $A=\left(M^{4} / \rho_{\mathrm{Ch} 0}\right)^{(\alpha+1)}$. We must impose $\alpha>-1$ for the accelerated epoch to occur after the decelerated phase. We also require $0<A<1$ so that $\rho_{\mathrm{Ch}}$ is well-defined for all times. Under these circumstances, at earlier times when $a / a_{0} \ll 1$, we have $\rho_{\text {Ch0 }} \propto a^{-3}$ and the fluid behaves as DM. For later times, $a / a_{0} \gg 1$, so we have $p_{\mathrm{Ch} 0}=-\rho_{\mathrm{Ch} 0}=-M^{4}=$ const. as in the cosmological constant case. Thus, this equation of state interpolates between DM and DE, while the average energy density of the universe changes, as expected from the previous discussion. It includes the standard Chaplygin gas for $\alpha=1$ and the $\Lambda \mathrm{CDM}$ model for $\alpha=0$ as special cases .

We now proceed to study the interplay between the detectability of cosmic topology and the GCG parameters $A$ and $\alpha$.

\section{Detectability of topology in GCG cosmology}

The key point for a systematic and quantitative study of the detectability of cosmic topology is the comparison between the injectivity radius of each manifold in units of the curvature radius $r_{\text {inj }}^{\mathrm{M}}$ (a topological invariant) and the survey depth $\chi_{\text {obs. }}$. As was pointed out in the previous section, the manifold's topology is detectable in principle for a survey of depth $\chi_{\text {obs }}$ if the matter-energy content parameters are such that $\chi_{\mathrm{obs}} \geq r_{\mathrm{inj}}^{\mathrm{M}}$. Likewise, if $\chi_{\mathrm{obs}}<r_{\text {inj }}^{\mathrm{M}}$, then the topology is undetectable by any repeated patterns method. Most of the work done so far has focused on the $\Lambda$ CDM model (see, e.g., Mota et al. 2003), but here we extend this approach to GCG models.

For the $\Lambda \mathrm{CDM}$ case (equivalent to $\alpha=0$ in the GCG model), the detectability conditions can be restated in terms of contour curves in the $\Omega_{\mathrm{m}}-\Omega_{\Lambda}$ parametric plane. For each manifold $M$ and a given fixed redshift $z_{\mathrm{obs}}$, we can define the contour curve $\chi\left(\Omega_{\mathrm{m}}, \Omega_{\Lambda}, z_{\mathrm{obs}}\right)=r_{\mathrm{inj}}^{\mathrm{M}}$. This curve lies in either the positive or negative curvature semiplanes $\left(\Omega_{0}<1\right.$ and $\Omega_{0}>1$, respectively), depending on whether the manifold is spherical or hyperbolic. The contour curve divides its semiplane into a region where the topology is undetectable $\left(\chi_{\mathrm{obs}}<r_{\mathrm{inj}}^{\mathrm{M}}\right)$ and a region where the topology is detectable in principle $\left(\chi_{\mathrm{obs}} \geq r_{\mathrm{inj}}^{\mathrm{M}}\right)$. Therefore, given this curve, it is possible to determine the (un)detectability of any given nonflat manifold for a range of density parameters.

In a previous work (Mota et al. 2003), it was shown that this contour curve can be approximated in two complementary ways by the tangent and secant lines, which respectively overestimate and underestimate detectability. It was further shown that the numerical results from both methods are in good agreement with each other, for the bounds on the density parameters obtained by Spergel et al. (2003), which means they are good approximations for the contour curve. Finally, it was also shown that, in the limit $z \rightarrow \infty$, the secant line can be obtained analytically. This last result is of particular interest, because it allows the study of the detectability of topology not only for individual manifolds, but also for whole classes of manifolds.

While in the $\Lambda$ CDM case the detectability was determined by two parameters, in the case of the GCG three parameters must be taken into account, $\Omega_{\mathrm{Ch} 0}=\rho_{\mathrm{Ch} 0} / \rho_{\text {crit }}, A$, and $\alpha$ (with the baryonic density $\Omega_{\mathrm{b} 0}$ kept fixed). To make the analysis simpler and comparisons easier, we introduce the new variables

$\bar{\Omega}_{\Lambda 0}=\Omega_{\mathrm{Ch} 0} A$,

$\bar{\Omega}_{\mathrm{m} 0}=\Omega_{\mathrm{Ch} 0}(1-A)$,

such that $\Omega_{\mathrm{Ch} 0}=\bar{\Omega}_{\mathrm{m} 0}+\bar{\Omega}_{\Lambda 0}$. Notice that with this definition, for $\alpha=0, \bar{\Omega}_{\mathrm{m} 0}$ corresponds to a matter density parameter and $\bar{\Omega}_{\Lambda 0}$ corresponds to a cosmological constant parameter (see Eq. (8) below).

With the definitions (7), the redshift-distance relation (4) becomes

$$
\begin{aligned}
\chi_{\mathrm{obs}}= & \sqrt{\left|1-\Omega_{0}\right|} \\
& \times \int_{0}^{z} \mathrm{~d} x\left\{\Omega_{\mathrm{Ch}}+\Omega_{\mathrm{b} 0}(1+x)^{3}+\left(1-\Omega_{0}\right)(1+x)^{2}\right\}^{-\frac{1}{2}},
\end{aligned}
$$

where

$\Omega_{\mathrm{Ch}}=\Omega_{\mathrm{Ch} 0}\left[\frac{\bar{\Omega}_{\Lambda 0}}{\Omega_{\mathrm{Ch} 0}}+\frac{\bar{\Omega}_{\mathrm{m} 0}}{\Omega_{\mathrm{Ch} 0}}(1+x)^{3(1+\alpha)}\right]^{\frac{1}{1+\alpha}}$

and $\Omega_{0}=\Omega_{\mathrm{Ch} 0}+\Omega_{\mathrm{b} 0}$.

In the analytical computations that follow, we omit the baryon fraction. The role of $\Omega_{\mathrm{b} 0}$ will be discussed later in conjunction with the numerical computations. Thus, for a fixed $z_{\mathrm{obs}}$ the only free parameters left are $\alpha, \bar{\Omega}_{\mathrm{m} 0}$, and $\bar{\Omega}_{\Lambda 0}$. For each value of $\alpha$, we can then define a parametric plane $\bar{\Omega}_{\mathrm{m} 0}-\bar{\Omega}_{\Lambda 0}$, which is very similar to the $\Omega_{\mathrm{m} 0}-\Omega_{\Lambda 0}$ plane discussed above. Unfortunately, it is not possible to analytically integrate Eq. (8) for a generic value of $\alpha$. Instead, we first show that $\chi_{\mathrm{obs}}$ is a monotonically decreasing function of $\alpha$ for $\alpha \in(-1, \infty)$, and then obtain analytical expressions for the contour curves for the limiting cases $\alpha=-1$ and $\alpha \rightarrow \infty$, with $z_{\mathrm{obs}} \rightarrow \infty$. This sets upper and lower bounds on the values that $\chi_{\text {obs }}$ can take in Eq. (8), and guarantees that $\chi_{\mathrm{obs}}$ will interpolate monotonically between these bounds for the intermediate values of $\alpha$.

Let us first show the decreasing monotonicity of $\chi_{\text {obs }}$ with respect to $\alpha$. Consider the term $\Omega_{\mathrm{Ch}}$ given by expression (9), with $x \geq 0$ and $\bar{\Omega}_{\Lambda 0} \neq 0$. Defining $u=(1+x)^{3(1+\alpha)} \geq 1$, a straightforward calculation shows that

$\frac{\partial^{2} \Omega_{\mathrm{Ch}}}{\partial u \partial \alpha}=\frac{\bar{\Omega}_{\mathrm{m} 0}}{\Omega_{\mathrm{Ch} 0}} \log \left[\frac{u}{\frac{\bar{\Omega}_{\Lambda}}{\Omega_{\mathrm{Ch} 0}}+\frac{\bar{\Omega}_{\mathrm{m}}}{\Omega_{\mathrm{Ch} 0}} u}\right] \geq 0$.

The derivative $\partial \Omega_{\mathrm{Ch}} / \partial \alpha$ is therefore an increasing function of $u$. But for $u=1$, clearly $\partial \Omega_{\mathrm{Ch}} / \partial \alpha=0$. Hence, $\partial \Omega_{\mathrm{Ch}} / \partial \alpha>$ 0 for any $u>1$, and $\Omega_{\mathrm{Ch}}$ is an increasing function of $\alpha$. Thus, from Eq. (8) we find that $\chi_{\text {obs }}$ is a decreasing function of $\alpha$. As an important result, the detectability of a given topology then becomes less likely as $\alpha$ increases, for fixed $\bar{\Omega}_{\mathrm{m}}$ and $\bar{\Omega}_{\Lambda}$. 
We now obtain analytical expressions for the contour curves in the extreme cases $\alpha=-1$ and $\alpha=\infty$ (with $\Omega_{\mathrm{b} 0}=0$ ). In both cases we take the $z_{\text {obs }} \rightarrow \infty$ limit, which is an upper bound to the size of the observable universe and does not give rise to significant numerical differences from $z_{\mathrm{obs}}=1089$, which corresponds to the last scattering surface.

When we take the $\alpha \rightarrow \infty$ limit in Eq. (9), it is clear that $\chi_{\mathrm{obs}}$ becomes a function of $\Omega_{\mathrm{Ch} 0}$ alone. We then solve the equation $\chi_{\mathrm{obs}}\left(\Omega_{\mathrm{Ch} 0}\right)=r_{\text {inj }}$ for $\Omega_{\mathrm{Ch} 0}$ to obtain

$$
\begin{array}{ll}
\bar{\Omega}_{\mathrm{m} 0}+\bar{\Omega}_{\Lambda 0}=\operatorname{sech}^{2}\left(\frac{r_{\mathrm{inj}}}{2}\right), & \text { for } \quad \Omega_{\mathrm{Ch} 0}<1, \\
\bar{\Omega}_{\mathrm{m} 0}+\bar{\Omega}_{\Lambda 0}=\sec ^{2}\left(\frac{r_{\mathrm{inj}}}{2}\right), & \text { for } \quad \Omega_{\mathrm{Ch} 0}>1 .
\end{array}
$$

$$
(\alpha \rightarrow \infty)
$$

The introduction of a small non-zero value of $\Omega_{\mathrm{b} 0}$ does not change this result significantly. The undetectability condition derived from the contour curves (11) can be stated as: for $\alpha \rightarrow \infty$ the manifold $M$ with injectivity radius $r_{\mathrm{inj}}^{\mathrm{M}}$ is undetectable, if either

$\bar{\Omega}_{\mathrm{m} 0}+\bar{\Omega}_{\Lambda 0}>\operatorname{sech}^{2}\left(\frac{r_{\mathrm{inj}}^{\mathrm{M}}}{2}\right)$ and $\Omega_{\mathrm{Ch} 0}<1$,

or

$\bar{\Omega}_{\mathrm{m} 0}+\bar{\Omega}_{\Lambda 0}<\sec ^{2}\left(\frac{r_{\mathrm{inj}}^{\mathrm{M}}}{2}\right)$ and $\Omega_{\mathrm{Ch} 0}>1$.

The calculation in the limit $\alpha \rightarrow-1$ is somewhat more involved. Let $\epsilon=\alpha-1$. We have, up to first order in $\epsilon$,

$$
\begin{aligned}
\Omega_{\mathrm{Ch}} & =\Omega_{\mathrm{Ch} 0}\left[\frac{\bar{\Omega}_{\Lambda 0}}{\Omega_{\mathrm{Ch} 0}}+\frac{\bar{\Omega}_{\mathrm{m} 0}}{\Omega_{\mathrm{Ch} 0}}(1+x)^{3 \epsilon}\right]^{1 / \epsilon}, \\
& =\Omega_{\mathrm{Ch} 0}\left[1+\frac{\bar{\Omega}_{\mathrm{m} 0}}{\Omega_{\mathrm{Ch} 0}} 3 \log (1+x) \epsilon\right]^{1 / \epsilon} .
\end{aligned}
$$

We can now take the limit $\epsilon \rightarrow 0$ in the above equation, substitute in Eq. (8), integrate, and solve for $\Omega_{\mathrm{Ch} 0}$ to finally obtain

$$
\begin{aligned}
& \bar{\Omega}_{\mathrm{m} 0}+\bar{\Omega}_{\Lambda 0}=\sec ^{2}\left(K r_{\mathrm{inj}}\right)+\tan ^{2}\left(K r_{\mathrm{inj}}\right) \bar{\Omega}_{\Lambda 0}, \\
& \text { for } \Omega_{\mathrm{Ch} 0}<1 \text { and } K>0, \\
& \bar{\Omega}_{\mathrm{m} 0}+\bar{\Omega}_{\Lambda 0}=\operatorname{sech}^{2}\left(K r_{\mathrm{inj}}\right)-\tanh ^{2}\left(K r_{\mathrm{inj}}\right) \bar{\Omega}_{\Lambda 0}, \\
& \bar{\Omega}_{\mathrm{m} 0}+\bar{\Omega}_{\Lambda 0}=1, \quad \text { for } \quad \Omega_{\mathrm{Ch} 0}>1 \text { and } K>0,
\end{aligned}
$$

$$
(\alpha=-1)
$$

where $K=\left(3 \bar{\Omega}_{\mathrm{m} 0} / 2 \Omega_{\mathrm{Ch} 0}-1\right)$. Here again, as well as in Eq. (15) below, the undetectability conditions can be stated from the corresponding equations of the contour curves by replacing the equalities by inequalities, as in (11) and (12). We note that, for current observational values, $K \leq 0$, and thus, in the limit $\alpha \rightarrow-1$, any non-flat topology would be detectable in principle. This is a consequence of the fact that in this case the contour curve and the flat line $\Omega_{\mathrm{Ch} 0}=1$ coincide (see Fig. 1), so there is no undetectable region. As we show, however, even

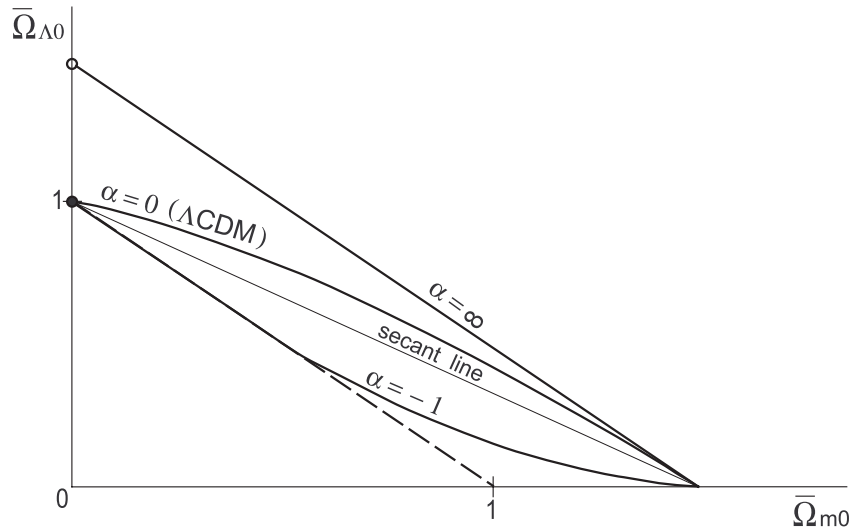

Fig. 1. Schematic contour curves $\chi_{\mathrm{obs}}=r_{\mathrm{inj}}^{\mathrm{M}}$ for $\alpha=-1,0$ and $\infty$, with $z_{\mathrm{obs}}=\infty$ and $\Omega_{\mathrm{b} 0}=0$. The curves for intermediate values of $\alpha$ interpolate monotonically between these extremes. Using $z_{\mathrm{obs}}=1089$ instead does not change the curves significantly. The presence of baryonic matter prevents the $\alpha=-1$ curve from touching the flat line (dashed).

a small non-zero value of $\Omega_{\mathrm{b} 0}$ renders many topologies unobservable.

Finally, as shown by Mota et al. (2003), the contour curve for the $\alpha=0$ (or $\Lambda \mathrm{CDM}$ ) case can be approximated well by the secant line, which is given by

$$
\begin{gathered}
\bar{\Omega}_{\mathrm{m} 0}+\bar{\Omega}_{\Lambda 0}=\operatorname{sech}^{2}\left(\frac{r_{\mathrm{inj}}}{2}\right)+\tanh ^{2}\left(\frac{r_{\mathrm{inj}}}{2}\right) \bar{\Omega}_{\Lambda 0} \text { for } \Omega_{\mathrm{Ch} 0}<1 \\
\bar{\Omega}_{\mathrm{m} 0}+\bar{\Omega}_{\Lambda 0}=\sec ^{2}\left(\frac{r_{\mathrm{inj}}}{2}\right)-\tan ^{2}\left(\frac{r_{\mathrm{inj}}}{2}\right) \bar{\Omega}_{\Lambda 0} \quad \text { for } \Omega_{\mathrm{Ch} 0}>1 . \\
(\alpha=0)
\end{gathered}
$$

Figure 1 illustrates the detectability schematically using contour curves in the parametric plane $\bar{\Omega}_{\mathrm{m} 0}-\bar{\Omega}_{\Lambda 0}$. We portray the contour curves for a spherical manifold associated with $\alpha=-1,0$, and $\infty$, as given above, as well as the secant line. For each of these cases, if $\bar{\Omega}_{\mathrm{m} 0}$ and $\bar{\Omega}_{\Lambda 0}$ take values between the respective contour curve and the flat line (dashed), then the topology of $M$ is undetectable.

For intermediate values of $\alpha$, we must turn to numerical integration to compute $\chi_{\mathrm{obs}}$ and quantitatively study the effects of the GCG parameters on the detectability of the topology. We consider the set of topologies presented in Tables 1 and 2, and assess their (un)detectability based on current observational values of the cosmological density parameters and bounds on the parameter $\alpha$ of the GCG. We are particularly interested in the effect of the value of $\alpha$ on the potential detectability of the topology, as different values of $\alpha$ can be thought of as different models for the matter-energy content.

In the numerical computations, we set $\Omega_{\mathrm{b} 0}=0.04$ in Eq. (8). This value can be obtained from observations of light element abundances, combined with primordial Big-Bang nucleosynthesis analysis (Burles et al. 2001; Kirkman et al. 2003) and the value of the Hubble constant (Freedman 2001). For $\Omega_{0}$ we use the limits from a combination of CMBR and largescale structure data obtained by Tegmark et al. (2004) for the $\Lambda \mathrm{CDM}$ case, $0.99<\Omega_{0}<1.03$. Finally we fix the total matter-like density parameter at $\bar{\Omega}_{\mathrm{m} 0}+\Omega_{\mathrm{b} 0}=0.3$. Our results, 

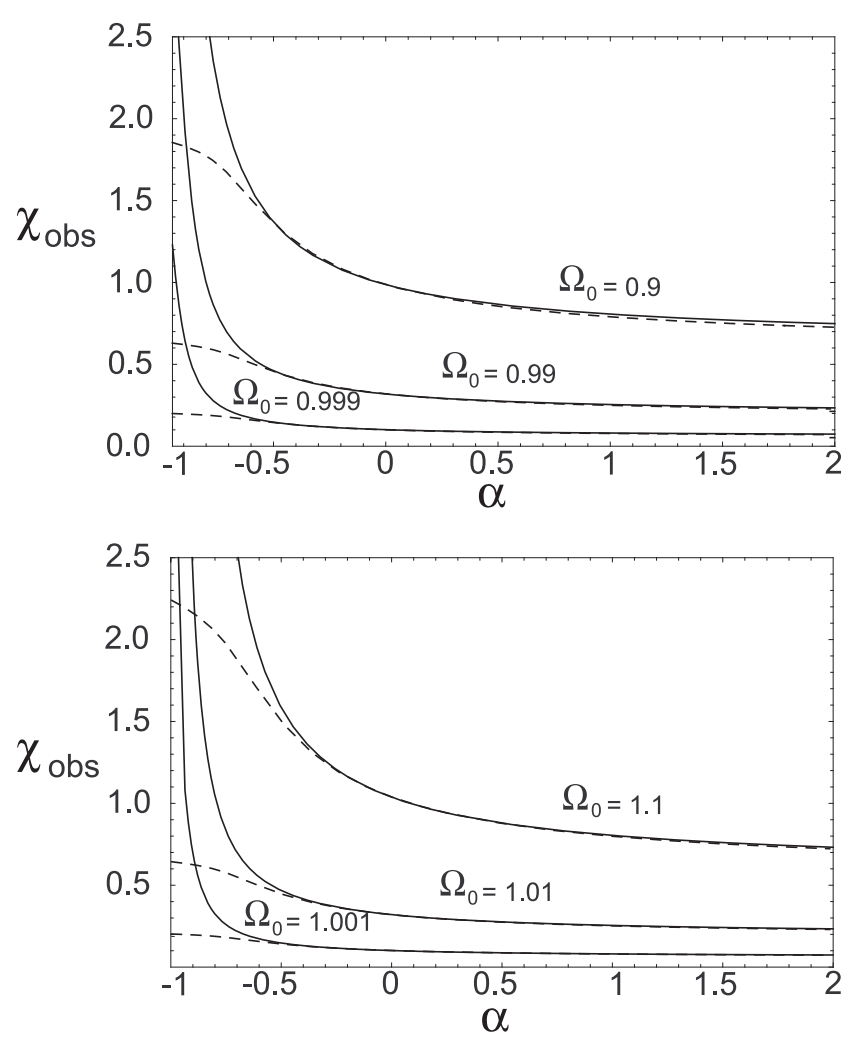

Fig. 2. Survey depth $\chi_{\mathrm{obs}}$, for several values of $\Omega_{0}=\Omega_{\mathrm{Ch} 0}+\Omega_{\mathrm{b} 0}$, for hyperbolic (top) and spherical (bottom) universes, with $z_{\mathrm{obs}}=1089$, and $\bar{\Omega}_{\mathrm{m} 0}+\Omega_{\mathrm{b} 0}=0.3$. The baryonic term is neglected in the solid lines and taken into account in the dashed lines.

however, are not very sensitive to the precise value of $\bar{\Omega}_{\mathrm{m} 0}$. Indeed, for values of $\alpha$ that are not too negative $(\alpha>-0.5)$, we have $\Delta \chi_{\text {obs }} / \chi_{\text {obs }} \simeq 0.15 \Delta \bar{\Omega}_{\mathrm{m} 0} / \bar{\Omega}_{\mathrm{m} 0}$.

To quantify the dependency on $\alpha$ and $\Omega_{\mathrm{b} 0}$, we plot the depth $\chi_{\text {obs }}$ in Fig. 2 as a function of $\alpha$ for various values of $\Omega_{0}$. The plot with $\Omega_{\mathrm{b} 0}=0$ is shown in solid lines, and the plot with $\Omega_{\mathrm{b} 0}=0.04$ is given by the dashed lines. The plots make a number of points clear. First, the value of $\chi_{\text {obs }}$ is not very sensitive to $\alpha$ for $\alpha>0$. Moreover, the change is very small for $\alpha>1\left(\Delta \chi_{\mathrm{obs}} / \chi_{\mathrm{obs}} \simeq 0.3\right.$ in the range $\left.1 \leq \alpha \leq \infty\right)$. This implies that the undetectability conditions (12), which were derived for $\alpha \rightarrow \infty$, are not much more restrictive than the undetectabilty condition obtained in the case of the standard Chaplygin gas (i.e., $\alpha=1$ ). Note also that the presence of a small $\Omega_{\mathrm{b} 0}$ component (dashed-line curves) does not change this picture in a significant way for positive values of $\alpha$. On the other hand, $\chi_{\mathrm{obs}}$ changes substantially when $\alpha<0$. This was expected, since we have shown that if $\Omega_{\mathrm{b} 0}=0$, then $\chi_{\mathrm{obs}}$ diverges in the limit $z \rightarrow \infty$ when $\alpha=-1$. The presence of a small amount of baryonic matter prevents this divergence (see the dashed-lines curves), although $\chi_{\text {obs }}$ still changes appreciably for $\alpha<0$. It is clear then that for sufficiently negative values of $\alpha$, the precise value of $\Omega_{\mathrm{b} 0}$ plays an important role in the detection of cosmic topology in the GCG context.

In Table 3 we display the manifolds from Tables 1 and 2, along with their respective injectivity radii. The upper (for spherical manifolds) and lower (for hyperbolic ones) bounds on $\Omega_{0}$ so that the manifold's topology is detectable in
Table 3. Minimum (maximum) values of $\Omega_{0}$ for each spherical (hyperbolic) topology to be potentially detectable (for $\bar{\Omega}_{\mathrm{M}}=0.26, \Omega_{\mathrm{b}}=$ 0.04 , and $z=1089$ ). Numbers in boldface indicate undetectabilty for the corresponding topologies.

\begin{tabular}{lccccc}
\hline \hline Manifolds & $r_{\text {inj }}$ & $\alpha=-1$ & $\alpha=-0.5$ & $\alpha=0$ & $\alpha=1$ \\
\hline$D_{9}^{*}, Z_{18}$ & $\frac{\pi}{18}$ & 1.001 & 1.002 & 1.003 & 1.004 \\
$D_{6}^{*}, Z_{12}$ & $\frac{\pi}{12}$ & 1.002 & 1.004 & 1.007 & 1.010 \\
$I^{*}, D_{5}^{*}, Z_{10}$ & $\frac{\pi}{10}$ & 1.003 & 1.005 & 1.010 & 1.015 \\
$O^{*}, D_{4}^{*}, Z_{8}$ & $\frac{\pi}{8}$ & 1.004 & 1.008 & 1.015 & 1.023 \\
$T^{*}, D_{3}^{*}, Z_{6}$ & $\frac{\pi}{6}$ & 1.007 & 1.014 & 1.026 & $\mathbf{1 . 0 4 2}$ \\
$D_{2}^{*}, Z_{4}$ & $\frac{\pi}{4}$ & 1.016 & $\mathbf{1 . 0 3 1}$ & $\mathbf{1 . 0 5 9}$ & $\mathbf{1 . 0 9 4}$ \\
\hline $\mathrm{m} 004(1,2)$ & 0.183 & 0.999 & 0.998 & 0.997 & 0.995 \\
$\mathrm{~m} 004(6,1)$ & 0.240 & 0.998 & 0.997 & 0.994 & 0.991 \\
$\mathrm{~m} 003(-4,3)$ & 0.288 & 0.998 & 0.996 & 0.992 & $\mathbf{0 . 9 8 7}$ \\
$\mathrm{m} 003(-2,3)$ & 0.289 & 0.998 & 0.996 & 0.992 & $\mathbf{0 . 9 8 7}$ \\
$\mathrm{m} 003(-3,1)$ & 0.292 & 0.998 & 0.996 & 0.992 & $\mathbf{0 . 9 8 7}$ \\
$\mathrm{m} 009(4,1)$ & 0.387 & 0.996 & 0.992 & $\mathbf{0 . 9 8 5}$ & $\mathbf{0 . 9 7 7}$ \\
$\mathrm{m} 007(3,1)$ & 0.416 & 0.995 & 0.991 & $\mathbf{0 . 9 8 3}$ & $\mathbf{0 . 9 7 4}$ \\
\hline
\end{tabular}

principle are also shown in the table for some noteworthy values of $\alpha$. We take the theoretical lower bound $\alpha=-1$; a numerical lower bound $\alpha=-1 / 2$ obtained from combining several observables and assuming a flat universe (see Makler et al. 2003b and Zhu 2004); the $\Lambda$ CDM case $\alpha=0$; and the standard Chaplygin Gas $\alpha=1$. The manifolds that are undetectable for $0.99 \leq \Omega_{0} \leq 1.03$ are indicated with boldface.

The table shows that the choice of $\alpha$ plays an important role in the detectability of the topology of these manifolds. In particular, negative values of $\alpha$ favor detectability. All the manifolds above are potentially detectable for $\alpha=-1$, and all but $D_{2}^{*}$ and $Z_{4}$ are for $\alpha=-1 / 2$. In the case of $\alpha=1$, most of the selected hyperbolic and many of the single action spherical manifolds are undetectable. From this table it is clear that the detectability of individual topologies depends significantly on the value of $\alpha$ and, in particular, may differ a lot from the case of a $\Lambda C D M$ model. This is an important point, inasmuch as it explicitly shows the dependence of the detectability with respect to the model for the matter-energy content.

Of course one cannot list all the (infinite) manifolds in the dihedral and cyclic groups. These two classes are particularly important, because as $\Omega_{0} \rightarrow 1$ from above, there is always an $n_{*}\left(\right.$ or $\left.m_{*}\right)$ such that the topology corresponding to $Z_{n}$ (or $D_{m}^{*}$ ) is detectable in principle for $n>n_{*}$ (or $m>m_{*}$ ). We have seen that (11) provides a lower bound for $\chi_{\mathrm{obs}}$ as a function of $\alpha$ and is also a fair approximation of the contour curves when $\alpha \geq 1$ for these manifolds. After solving (11) for $n_{*}$ and $m_{*}$, we can state that in a dihedral or lens space, for any value of $\alpha$ the 
topology is detectable (in principle) if

$$
\begin{aligned}
& n_{*} \geq \operatorname{int}\left[\frac{\pi}{2} \frac{1}{\arctan \left(\sqrt{\Omega_{\mathrm{Ch} 0}-1}\right)}\right], \\
& m_{*} \geq \operatorname{int}\left[\pi \frac{1}{\arctan \left(\sqrt{\Omega_{\mathrm{Ch} 0}-1}\right)}\right],
\end{aligned}
$$

where int denotes the integer part of the argument. More specifically, for $\alpha \geq 1$ these are approximately equalities, since as can be seen in Fig. $2, \chi_{\text {obs }}$ is not very sensitive to the value of $\alpha$ for $\alpha \geq 1$. Recall that, although these expressions do not take baryons into account, their contribution is negligible for $\alpha \geq 1$. Thus, Eqs. (16) provide an absolute lower bound for the detectability of cyclic and dihedral topologies. Any values of $n(m)$ greater than $n_{*}\left(m_{*}\right)$ will render the topology potentially detectable for any value of $\alpha$.

Given the increasing amount of high quality cosmological data, in particular the availability of high resolution full sky CMBR maps (see Spergel et al. 2003), we can expect to detect a non-trivial topology, if it is present and if $r_{\text {inj }}<\chi_{\text {obss }}$. This can be done, for example, by observing matching circles in the CMBR maps. In this regard, some topological signatures in CMBR data have recently been proposed (see Luminet et al. 2003; Cornish et al. 2004; Roukema et al. 2004; Aurich et al. 2005a, 2005b). One can then ask whether the hypothetical detection of a nontrivial topology may lead to better knowledge of the cosmological model. In the case of the GCG, does the determination of a given topology impose any constraint on $\alpha$ ?

The answer is positive, as can be seen from Fig. 3, where we plot the contour curves $\chi_{\mathrm{obs}}\left(\Omega_{0}, \alpha\right)=r_{\text {inj }}^{\mathrm{M}}$ in the $\Omega_{0}-\alpha$ parametric plane for the topologies discussed in the previous section, where we fix $z_{\mathrm{obs}}=1089, \Omega_{\mathrm{b} 0}=0.04$, and $\bar{\Omega}_{\mathrm{m} 0}=0.26$. Recall from Sect. 3 that each such contour curve separates the parametric plane into two regions where the manifold in question is either potentially detectable or undetectable. It is clear, for example, that the detection of a binary tetrahedral topology $\left(T^{*}\right)$ would place the constraint $\alpha<0.2$ for current bounds on $\Omega_{0}$. If future observations tighten the range of $\Omega_{0}$ even closer to 1 , say $\Omega_{0}=1.000 \pm 0.003$, the detection of either $D_{9}^{*}, Z_{18}$, m004 $(1,2)$, or m004 $(6,1)$ would imply $\alpha \lesssim-0.5$. Figure 3 shows that a topology that would be detectable in a $\Lambda$ CDM universe is not necessarily ruled out, if no observational topological signature is found. For example, if no sign is detected of the binary octahedral $\left(O^{*}\right)$ topology in CMBR maps, it could simply be that $\alpha \gtrsim 2.5$.

\section{Discussion and concluding remarks}

We have considered the detectability of a non-trivial cosmic topology in a universe dominated by the GCG. This component offers the possibility of DM/DE unification and is consistent with a number of cosmological observations. We investigated how the detectability is altered with respect to changes in the model parameters, as well as to the choice of the model itself, using the GCG family as a concrete example.

In the case of the $\Lambda \mathrm{CDM}$ model, the detectablity of a manifold's topology is a function of the component densities, $\Omega_{\mathrm{m} 0}$ and $\Omega_{\Lambda 0}$. Consideration of the GCG brings a new parameter

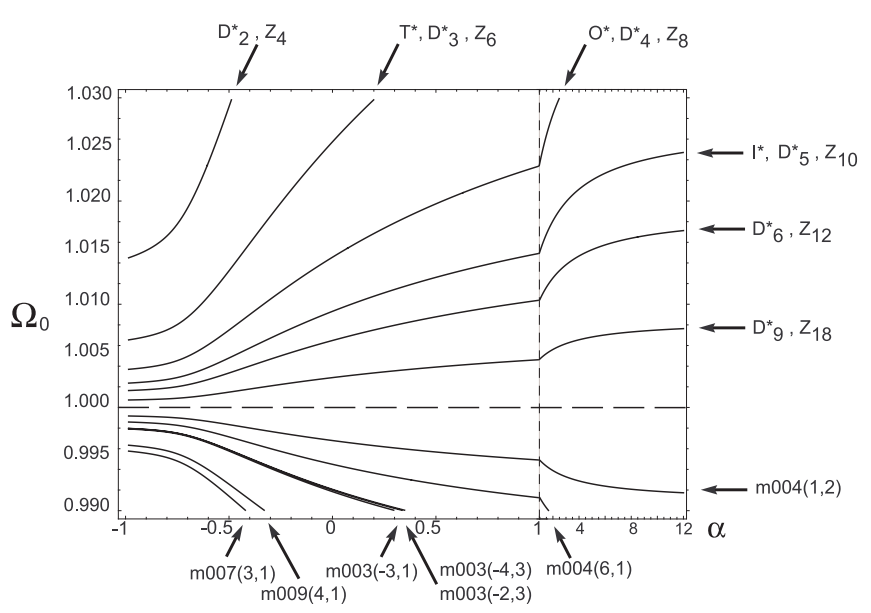

Fig. 3. Curves of constant $\chi_{\mathrm{obs}}=r_{\mathrm{inj}}^{\mathrm{M}}$, in the $\left(\Omega_{0}, \alpha\right)$ plane for the same topologies as in Table 3. The $\alpha$ axis is compressed for $1 \leq \alpha \leq 12$. Again we fix $z=1089$, and $\bar{\Omega}_{\mathrm{m} 0}+\Omega_{\mathrm{b} 0}=0.3$, with $\Omega_{\mathrm{b} 0}=0.04$.

into the analysis, the steepness of the equation of state $\alpha$. We have shown that, for fixed $\Omega_{0}$ and $\bar{\Omega}_{\mathrm{m} 0}$, more topologies become potentially detectable as $\alpha$ decreases. We have obtained analytical results for $\alpha \rightarrow \infty$ to establish a lower bound for the detectability in the GCG case. In general, the detectability is not very sensitive to $\alpha$, for $\alpha>0$, but it varies greatly for negative values of $\alpha$. In this case, the contribution of baryons is important and must be taken into account. For example, we showed that for $\alpha=-1$, any topology would be potentially detectable if we neglect $\Omega_{\mathrm{b} 0}$. However, considering the baryonic contribution, many manifolds would still be unobservable.

Detectable non-trivial topologies leave an imprint on the CMBR that are expected to be measurable in current and forthcoming maps. Detection of a cosmic topology is therefore a realistic possibility in the near future, so we investigated what constraints would be imposed on the parameters of the model by such a detection. For example, hypothetical detection of a binary tetrahedral topology would place the constraint $\alpha<0.2$ for $\Omega_{0}<1.03, \bar{\Omega}_{\mathrm{m} 0}=0.26$, and $\Omega_{\mathrm{b}}=0.04$. A more thorough study of the inverse problem has been developed in Makler et al. (2005).

We stress that the method discussed here for studying the detectability of cosmic topology can be applied to other models with different expansion histories of the universe. In fact, the impact on the detectability appears through the ratio $H_{0} / H(z)$ (cf. Eq. (4)), which in turn depends on the choice of the model. For instance, the detectability could be studied in models of DE using generic parametrizations for its equation of state, such as the widely studied (linear in the scale factor) $p(a) / \rho(a)=$ $w(a)=w_{0}+w_{a}(1-a)$ (see, for instance, Chevallier \& Polarski 2001 and Linder 2003).

An important point that emerges from these results is that, given a set of observational constraints, the detectability of cosmic topology depends on the choice of the cosmological model. Thus, any attempt to rule out a given topology must take this into account.

Acknowledgements. We thank MCT, CNPq, and FAPERJ for the grants under which this work was carried out, and the anonymous referee for relevant comments. 


\section{References}

Aurich, R., Lustig, S., \& Steiner, F. 2005a, Class. Quant. Grav., 22, 2061

Aurich, R., Lustig, S., \& Steiner, F. 2005b, Class. Quant. Grav., 22, 3443

Bento, M. C., Bertolami, O., \& Sen, A. A. 2002, Phys. Rev. D, 66, 043507

Bertone, G., Hooper, D., \& Silk, J. 2005, Phys. Rept., 405, 279

Bilić, N., Tupper, G. B., \& Viollier, R. D. 2002, Phys. Lett. B, 535, 17

Burles, S., Nollett, K. M., \& Turner, M. S. 2001, ApJ, 552, L1

Chevallier, M., \& Polarski, D. 2001, Int. J. Mod. Phys., D, 10, 213

Cornish, N. J., Spergel, D. N., \& Starkman, G. D. 1998, Class. Quant. Grav., 15, 2657

Cornish, N. J., Spergel, D. N., \& Starkman, G. D. 2004, Phys. Rev. Lett., 92, 201302

Dev, A., Jain, D., \& Alcaniz, J. S. 2004, A\&A, 417, 847

Ellis, G. F. R. 1971, Gen. Rel. Grav., 2, 7

Freedman, W. 2001, AJ, 553, 47

Gausmann, E., Lehoucq, R., Luminet, J.-P., Uzan, J.-P., \& Weeks, J. 2001, Class. Quant. Grav., 18, 5155

Gomero, G. I., , Rebouças, M. J., \& Tavakol, R. 2001a, Class. Quantum Grav., 18, 4461

Gomero, G. I., , Rebouças, M. J., \& Teixeira, A. F. F. 2001b, Class. Quantum Grav., 18, 1885

Hodgson, C. D., \& Weeks, J. R. 1994, Experimental Mathematics, 3, 261

Kamenshchik, A., Moschella, U., \& Pasquier, V. 2001, Phys. Lett. B, 511,265

Kirkman, D., Tytler, D., Suzuki, N. J., O’Meara, M., \& Lubin, D. 2003, ApJS, 149, 1

Lachièze-Rey, M., \& Luminet, J.-P. 1995, Phys. Rep., 254, 135

Lehoucq, R., Lachièze-Rey, M., \& Luminet, J.-P. 1996, A\&A, 313, 339

Levin, J. 2002, Phys. Rep., 365, 251

Linder, E. 2003, Phys. Rev. Lett., 90, 091301

Luminet, J.-P., Weeks, J., Riazuelo, A., Lehoucq, R., \& Uzan, J.-P. 2003, Nature, 425, 593
Makler, M. 2001 Gravitational Dynamics of Structure Formation in the Universe, Ph.D. Thesis, Brazilian Center for Research in Physics

Makler, M., Oliveira, S. Q., \& Waga, I. 2003a, Phys. Lett. B, 555, 1

Makler, M., Oliveira, S. Q., \& Waga, I. 2003b, Phys. Rev. D, 64, 123521

Makler, M., Mota, B., \& Rebouças, M. J. 2005 [arXiv: astro-ph/0507116]

Mota, B., Rebouças, M. J., \& Tavakol, R. 2003, Class. Quantum Grav., 20, 4837

Mota, B., Gomero, G. I., Rebouças, M. J., \& Tavakol, R. 2004, Class. Quantum Grav., 21, 3361

de Oliveira-Costa, A., Tegmark, M., Zaldarriaga, M., \& Hamilton, A. 2004, Phys. Rev. D, 69, 063516

Perlmutter, S., Aldering, G., della Valle, M., et al. 1998, Nature, 391, 51

Perrotta, F., Matarrese, S., \& Torki, M. 2004, Phys. Rev. D, 70, 121304

Rebouças, M. J., \& Gomero, G. I. 2004, Braz. J. Phys., 34, 1358 [arXiv: astro-ph/0402324]

Reis, R. R. R., Waga, I., Calvão, M. O., \& Jorás, S. E. 2003, Phys. Rev. D, 68, 061302

Riess, A. G., Filippenko, A. V., Challis, P., et al. 1998, AJ, 116, 1009

Roukema, B. F., Bartosz, L., Cechowska, M., Marecki, A., \& Bajtlik, S. 2004, A\&A, 423, 821

Spergel, D. N., Verde, L., Peiris, H. V., et al. 2003, ApJ Suppl., 148, 175

Starkman, G. D. 1998, Class. Quantum Grav., 15, 2529

Tegmark, M., et al. 2004, Phys. Rev. D, 69, 103501

Tonry, J. L., Schmidt, B. P., Barris, B., et al. 2003, AJ, 594, 1

Threlfall, W., \& Seifert, H. 1932, Math. Annalen, 104, 543

Uzan, J.-P., Lehoucq, R., \& Luminet, J.-P. 1999, A\&A, 351, 766

Weeks, J. R. 1999, SnapPea: A computer program for creating and studying hyperbolic 3-manifolds, available at http://geometrygames.org/SnapPea/

Weeks, J. R. 2003, Mod. Phys. Lett. A, 18, 2099

Weeks, J. R., Lehoucq, R., \& Uzan, J.-P. 2003, Class. Quant. Grav., 20,1529

Wolf, J. A. 1984, Spaces of Constant Curvature, fifth ed., Publish or Perish Inc., Delaware

Zhu, Z.-H. 2004, A\&A, 423, 421 